\title{
INÉGALITÉS MONDIALES ET CHANGEMENT CLIMATIQUE
}

\author{
Céline Guivarch, Nicolas Taconet \\ ENPC (École des Ponts ParisTech), CIRED
}

Dans cet article, nous synthétisons les travaux récents sur les liens entre climat et inégalités pour montrer comment les enjeux liés aux impacts et à l'atténuation du changement climatique affectent les inégalités, à la fois entre pays et entre individus. Dans un premier temps, nous analysons les inégalités d'exposition et de vulnérabilité aux impacts du changement climatique. Puis, nous nous intéressons aux inégalités dans la contribution aux émissions de gaz à effet de serre entre pays et entre individus. Dans un dernier temps, nous montrons comment les inégalités face au changement climatique permettent d'éclairer l'équité de la répartition des actions pour lutter contre le changement climatique.

Les dernières décennies ont été marquées par une certaine convergence économique entre pays, notamment portée par le développement rapide de l'Inde et de la Chine, bien que les taux de croissance du PIB demeurent bas dans certains pays africains (Firebaugh, 2015 ; Milanovic, 2016). En revanche, les inégalités de revenu à l'intérieur des pays ont eu tendance pendant la même période à augmenter (Alvaredo et al., 2018). Par exemple, aux États-Unis, les revenus des $10 \%$ les plus pauvres ont stagné depuis les années 1980 quand ceux des $1 \%$ les plus riches ont crû en moyenne de $2 \%$ par an (Thomas Piketty, Saez et Zucman 2018). En considérant à la fois les inégalités entre pays et à l'intérieur des pays, la croissance des revenus depuis 1990 a été très inégalement distribuée entre les différents déciles de revenus à l'échelle mondiale, ainsi que le montre la courbe 
dite de «l'éléphant» (Milanovic, 2016 ; Alvaredo et al., 2018). Aux deux extrémités de la distribution, les plus pauvres n'ont que peu bénéficié de cette croissance, tandis que les $1 \%$ les plus riches ont connu une forte croissance de leurs revenus. Entre les deux, l'augmentation des revenus d'une grande partie de la population dans les économies émergentes contraste avec le déclin de la classe moyenne des pays développés.

Dans le même temps, les émissions mondiales de gaz à effet de serre n'ont fait que croître, et on observe déjà un réchauffement moyen global de $1,1^{\circ} \mathrm{C}$ par rapport à l'ère pré-industrielle, ce qui a des conséquences importantes sur les inégalités de revenu. En effet, climat et inégalités sont étroitement liés, pour plusieurs raisons. Les conditions climatiques et écologiques dont jouissent les pays expliquent en partie les écarts dans leurs performances économiques (Mellinger, Sachs et Gallup, 2000). De plus, tant au niveau des pays qu'à celui des individus, ce sont en général les moins riches qui sont les plus vulnérables aux impacts du changement climatique. Les différents effets du changement climatique (vagues de chaleur, sécheresses, montée du niveau de la mer, ...) touchent de manière disproportionnée les moins riches. Ils risqueraient de ralentir la convergence espérée entre les pays et de rendre plus difficile la réduction des inégalités observée à l'intérieur des pays.

Par ailleurs, les inégalités économiques se reflètent dans les différences de contribution aux émissions de gaz à effet de serre à l'échelle globale. Les pays développés et les individus les plus riches, de par leur niveau de consommation, contribuent de manière disproportionnée à l'augmentation de la température. II y a là une double peine : ce sont ceux qui risquent de subir le plus les conséquences du changement climatique qui contribuent le moins au problème (Roberts, 2001 ; Althor, Watson et Fuller, 2016) (GIEC Rapport Spécial 1.5, Chapitre 3) et réciproquement, les pays les plus responsables qui sont aussi les moins vulnérables (Carte 1).

Enfin, la conception et la mise en œuvre des politiques climatiques pour réduire les émissions de gaz à effet de serre et pour s'adapter à un climat qui change reposent la question des inégalités entre pays et au sein des pays. Entre pays, elles soulèvent des enjeux d'équité de la répartition des actions d'atténuation, d'adaptation et de leur financement. Au sein des pays, les politiques climatiques peuvent induire des inégalités lorsque leurs coûts pèsent davantage sur les plus modestes 
Carte 1. Contribution et réception des impacts du réchauffement climatique

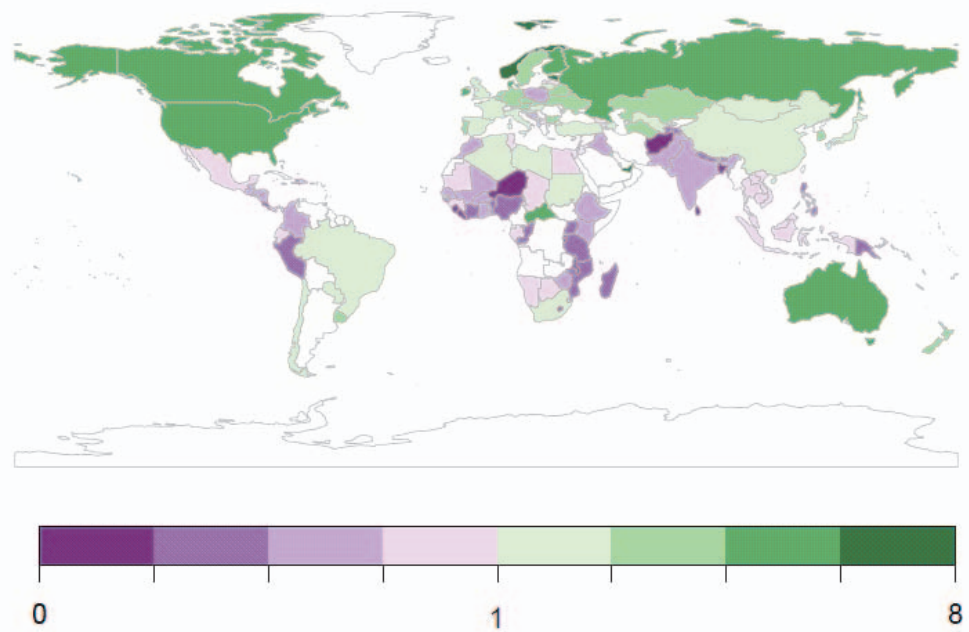

Note : Le ratio Émission-Émergence (échelle logarithmique) rapporte la contribution des émissions aux impacts à venir. Une valeur supérieure à 1 signifie qu'un pays est relativement plus responsable du réchauffement climatique qu'il n'en subira les impacts. Voir les Cartes 2 et 3 pour les deux composantes du ratio.

Source : Données de Frame et al., 2019.

ou lorsque certaines catégories sociales sont exclues de leurs bénéfices. Par exemple, les politiques d'atténuation ont des effets sur les prix de l'énergie ou de l'alimentation, avec un risque pour les plus pauvres de faire face à une baisse de leur niveau de vie, et pour les pays pauvres de ralentir leur développement (GIEC Rapport Spécial 1.5, Chapitre 5). À l'inverse, il s'agit de comprendre dans quelles conditions les politiques climatiques peuvent être conciliées avec l'atteinte d'objectifs de développement, de réduction de la pauvreté et des inégalités.

Dans cet article, nous synthétisons les travaux récents sur les liens entre climat et inégalités pour montrer comment les enjeux liés aux impacts et à l'atténuation du changement climatique affectent les inégalités, à la fois entre pays, et entre individus. Dans un premier temps, nous analysons les inégalités d'exposition et de vulnérabilité aux impacts du changement climatique. Puis, nous nous intéressons aux inégalités dans la contribution aux émissions de gaz à effet de serre entre pays et entre individus. Dans un dernier temps, nous montrons comment les inégalités face au changement climatique permettent d'éclairer l'équité de la répartition des actions pour lutter contre le changement climatique. 


\section{Encadré 1. Définir les inégalités}

L'étude des inégalités s'intéresse à la façon dont certains avantages sont distribués au sein d'une société (justice distributive) et au caractère juste des procédures de distribution de ces avantages (justice procédurale). Au sens économique du terme, on entend souvent par inégalités la dispersion des revenus entre individus au sein d'une population. On peut la mesurer à l'aide d'indicateurs tels que l'indice de Gini, qui mesure l'écart entre la distribution observée des revenus et une répartition égalitaire idéale où chacune possède exactement le même revenu. On peut également analyser la situation d'une proportion donnée des ménages les plus modestes et la mettre en regard de celle des plus riches. On mesure ainsi à quel point les revenus sont inégalement répartis entre individus ou entre pays. Les revenus donnent cependant une vision limitée des inégalités économiques : le patrimoine, à la fois foncier et financier, est souvent plus concentré que les revenus, et il constitue donc une source importante d'inégalités entre individus. Les inégalités de patrimoine ont dans l'ensemble augmenté au cours des dernières décennies et la part de la richesse détenue par les $1 \%$ les plus riches est passée de $28 \%$ en 1980 à $33 \%$ en 2017 (Alvaredo et al., 2018).

De plus, les inégalités ne se limitent pas aux aspects purement économiques et sont souvent multidimensionnelles (voir GIEC, Cinquième Rapport d'évaluation, Groupe 2, Chapitre 13). D'autres types d'inégalités sociales peuvent fortement influencer les conditions de vie des individus et les opportunités dont ils bénéficient (Crow, Zlatunich et Fulfrost, 2009 ; Sen, 1997), comme l'accès à la santé, à l'éducation, la participation à la prise de décision, ainsi que les inégalités liées à la race ou au genre, qui peuvent exclure des groupes sociaux de l'accès à des emplois, des services sociaux. Enfin les inégalités peuvent être de nature environnementale, par l'accès différencié à certaines ressources naturelles, services rendus par la nature, ou par l'exposition à des externalités de pollution.

\section{Encadré 2. Typologie des inégalités liées au changement climatique}

II existe différents types d'inégalités liées à l'environnement que l'on peut catégoriser ainsi (Laurent, 2011) :

- Les inégalités d'exposition et d'accès font référence à l'inégale distribution de la qualité de l'environnement entre différents groupes sociaux, qu'elle soit positive (accès à certaines aménités) ou négatives (exposition à la pollution). Dans le cas du changement climatique, les individus et les pays sont et seront inégalement affectés par ses conséquences (montée des eaux, événements extrêmes, ...) (cf. partie 1) ;

- Les inégalités d'impacts reflètent la contribution différenciée des individus à la dégradation de l'environnement, notamment dans les 
émissions de gaz à effet de serre responsables du changement climatique (cf. partie 2) ;

- Les inégalités induites par les politiques climatiques interviennent lorsque les actions d'atténuation ou d'adaptation amplifient les inégalités, par exemple leurs coûts peuvent davantage peser sur les ménages les plus modestes ou parce que certaines catégories peuvent être exclues de leurs bénéfices (cf. partie 3) ;

- Les inégalités dans l'accès à la décision proviennent du fait que certains individus ou groupes sociaux ont moins de pouvoir pour infléchir les décisions politiques.

\section{Les pays pauvres et les individus pauvres sont les plus vulnérables aux impacts du changement climatique}

Les inégalités au sein des populations existent en dehors de toute considération liée au changement climatique. De même que de nombreux facteurs liés aux institutions, à l'éducation, au marché du travail ou aux structures sociales, le climat joue un rôle dans les conditions de vie des individus, puisqu'il affecte certaines sources de revenus (notamment agricoles), qu'il peut entraîner des destructions d'habitats ou de capital physique et qu'il a un effet sur le bien-être et la santé. Tous les individus ne sont pas affectés de la même manière par le changement climatique: les impacts physiques seront différents $d^{\prime} u n e$ région à l'autre. De plus, les impacts économiques dépendent de la vulnérabilité socio économique des individus et des pays. D'une façon générale, les pays pauvres et les individus les plus modestes sont les plus vulnérables aux impacts du changement climatique : ils sont à la fois plus exposés, plus sensibles à ces impacts et ont une moins grande capacité d'adaptation (Carte 2). Le changement climatique exacerbe déjà et risque d'exacerber encore davantage les inégalités existantes.

Les impacts physiques sont déjà visibles et seront plus importants dans les pays pauvres (GIEC, Rapport Spécial 1.5, Chapitre 3). Du fait de leur localisation, les pays pauvres sont les plus exposés aux différents effets du changement climatique, que ce soit le stress hydrique, l'intensité des sécheresses ou les vagues de chaleur, les pertes de rendements agricoles ou la dégradation des habitats naturels. On estime ainsi, en utilisant des indicateurs prenant en compte ces effets du changement climatique, que l'exposition aux risques climatiques porte à environ $90 \%$ sur l'Afrique et l'Asie du Sud-Est (Byers et al., 2018), et ce sont les individus les plus pauvres à l'intérieur de ces régions qui sont les plus à risque. 
Carte 2. Indice de vulnérabilité des pays au réchauffement climatique
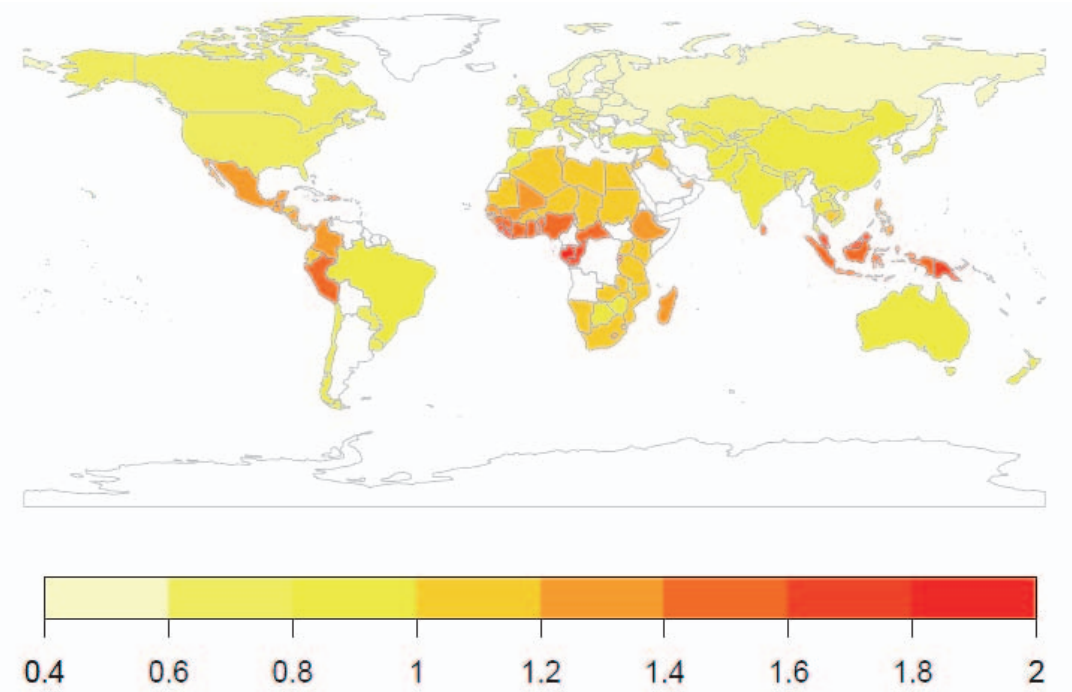

Note : L'indice de vulnérabilité des pays au réchauffement climatique utilise le ratio « signal-sur-bruit » normalisé. Le ratio permet de rapporter l'augmentation de température à venir par rapport à la variabilité historique observée dans le pays, et renseigne sur la sensibilité au changement climatique.

Source : Données de Frame et al., 2019.

Pour le secteur agricole, les études montrent que les impacts du changement climatiques sont négatifs dans l'ensemble, en particulier dans les régions de basse latitude dans lesquelles les pays en développement sont concentrés (Rosenzweig et al., 2014). Cet effet différencié entre les pays s'observe déjà : bien que le changement climatique a diminué les rendements agricoles dans la plupart des régions (Lobell, Schlenker et Costa-Roberts, 2011), certains pays développés notamment en Europe, ont bénéficié de ce réchauffement, par exemple le Royaume-Uni (Jaggard, Qi et Semenov, 2007), l'Écosse (Gregory et Marshall, 2012) et d'autres pays d'Europe du Nord (Supit et al., 2010).

Différents indicateurs permettent également d'illustrer cette distribution inégalitaire des impacts physiques. Les extrêmes de température journaliers attendus du fait du changement climatique sont localisés dans des zones moins développées (Harrington et al., 2016). S'il existe une incertitude à l'échelle globale sur l'évolution des ressources en eau du fait du changement climatique, les régions dans lesquelles on estime que le stress hydrique augmentera sont des zones défavorisées, en particulier dans le Nord de I'Afrique (Gosling et Arnell, 2016). Ces impacts différenciés selon les pays touchent aussi les écosystèmes qu'ils abritent. 
Les écosystèmes tropicaux sont souvent adaptés à d'étroites conditions écologiques quand ceux des zones tempérées peuvent s'adapter à des variations plus importantes de climat qu'ils connaissent au cours de l'année. Les écosystèmes tropicaux sont donc menacés pour de plus faibles variations de température. Pour cette raison, la limitation de la température globale à $1.5^{\circ} \mathrm{C}$ plutôt qu'à $2^{\circ} \mathrm{C}$ bénéficierait aux pays les plus pauvres (King et Harrington, 2018).

Au sein des pays, les communautés ou ménages pauvres sont également localisés dans des zones à plus fort risque climatique, pour lequel le foncier est souvent plus abordable ou parce qu'ils offrent des opportunités en termes d'accès à l'emploi, d'éducation ou de santé. Ils peuvent être contraints de vivre dans des zones inondables ou dans les zones risquées des deltas (« World's 15 Countries with the Most People Exposed to River Floods», 2015 ; Brouwer et al., 2007). En ville, les habitats informels sont fréquemment situés dans des zones soumises à des aléas climatiques, par exemple à Dhaka (Braun et Aßheuer, 2011), ou sur des parcelles en pentes susceptibles de connaître des coulées de boue, notamment en Amérique du Sud (Painter, 2007). En particulier, les plus pauvres sont de manière disproportionnée localisés dans des zones qui présentent des risques d'inondations urbaines et de sécheresse, et le nombre de personnes exposées pourrait augmenter d'environ $10 \%$ en 2030 en l'absence de réduction d'émissions (Jongman et al., 2015). Il en va de même pour l'exposition aux extrêmes de chaleur attendus du fait du changement climatique car dans les pays chauds les plus modestes tendent à se concentrer dans des zones dans lesquelles les températures sont plus élevées (Park et al., 2018).

De plus, les mêmes impacts physiques ne se traduisent pas par les mêmes dommages, du fait d'une sensibilité et de capacités d'adaptation différentes entre pays et entre individus. La plus grande sensibilité des pays pauvres aux impacts du changement climatique s'explique notamment du fait de la place des secteurs agricole, forêts et pêche dans l'économie. Un part importante de la population dépend directement d'activités susceptibles d'être affectées par le changement climatique, en particulier les plus modestes dont la survie dépend davantage du capital naturel à portée de main que du capital physique ou humain (Huq et al., 2010), et qui bénéficient de nombreux services rendus par la nature (Noack et al., 2015), lesquels peuvent être menacés par le changement climatique. 
Les plus modestes sont aussi fortement vulnérables aux événements extrêmes comme les catastrophes naturelles qui risquent d'augmenter du fait du changement climatique. Leurs habitations sont de moindre qualité donc plus sensibles aux aléas climatiques. Les coûts de réparation cumulés peuvent représenter une part plus importante de leurs revenus que pour les foyers aisés, comme cela a été le cas à la suite des inondations de Bombay en 2005 (Patankar, 2015). Bien que le nombre de catastrophes naturelles entre pays à bas et hauts revenus soit équivalent depuis les années 1970, le nombre de morts est 10 fois plus élevé dans les pays les plus pauvres (Strömberg, 2007). Au-delà des revenus, les institutions jouent également un rôle important dans la protection des populations face aux catastrophes naturelles (Kahn, 2005). La différence de vulnérabilité entre pays riches et pauvres tend à diminuer mais reste encore considérable : pour la période 2007-2016, le taux de mortalité due aux catastrophes naturelles est environ 4 fois supérieur dans les pays pauvres (Formetta et Feyen, 2019).

\section{Encadré 3. L'ouragan Harvey}

Le cas de l'ouragan Harvey qui a frappé le Texas en 2017 montre que les pays développés sont aussi vulnérables aux événements climatiques extrêmes. L'ouragan et ses pluies torrentielles ont entraîné la mort d'une centaine de personnes et causé des dégâts estimés à une centaine de milliards de dollars. Ce sont les plus pauvres qui ont subi l'essentiel des dommages puisque les ménages modestes étaient concentrés dans des zones inondables (Reeves, 2017). II est également plus difficile de se relocaliser pour eux en cas de désastre (Boustan et al., 2017). La plupart d'entre eux ne bénéficiaient pas d'assurance, ce qui peut les faire basculer de manière durable dans la pauvreté.

D'après le GIEC, I'intensité des ouragans risque d'augmenter avec le changement climatique. En particulier, la probabilité annuelle d'occurrence au Texas d'une pluviosité comparable à l'ouragan Harvey augmenterait pour attendre $18 \%$ à la fin du XXI siècle dans le scénario de émissions de gaz à effet de serre le plus pessimiste, alors qu'elle n'était que de $1 \%$ pour la période 1980-2000 (Emanuel, 2017).

Enfin, ce sont les ménages les plus modestes qui risquent de subir les divers effets du changement climatique sur la santé, via les vagues de chaleur (Ahmadalipour, Moradkhani et Kumar, 2019) et la propagation de différentes maladies (malaria, dengue). Les vagues de chaleur touchent différemment les groupes sociaux. Dans le cas de la canicule 
européenne de 2003, au-delà du critère démographique $90 \%$ des victimes en France avaient plus de 65 ans (la mortalité a été plus grande pour les catégories sociales les moins élevées, Borrell et al., 2006). Les températures de cette canicule pourraient correspondre à un été moyen à la fin du siècle dans les scénarios d'émissions élevées.

Les plus modestes doivent également faire face à des impacts indirects, comme l'augmentation des prix des denrées agricoles qui résulte des moindres rendements ou d'événements météorologiques extrêmes (Hallegatte et Rozenberg, 2017). Ils sont particulièrement sensibles aux variations de ces prix puisqu'ils consacrent une part importante de leurs revenus à l'alimentation. La montée des prix pourrait menacer la sécurité alimentaire dans certaines régions, notamment en Afrique subsaharienne ou en Asie du Sud, ce qui augmenterait la pauvreté dans ces régions (Hertel, 2015). Un autre mécanisme impliquant des impacts indirects peut toucher les revenus lorsque la productivité du travail vient à diminuer du fait de fortes température (Deryugina et Hsiang, 2014 ; Heal et Park, 2016), en particulier pour du travail en extérieur.

Pour ces différents types d'impacts, la capacité d'adaptation des plus modestes est moins élevée et le changement climatique vient aggraver des difficultés préexistantes. La plupart du temps ils ne bénéficient pas de mécanismes assurantiels ou d'accès à des services de santé de base qui permettent d'atténuer des chocs sur les prix ou les revenus. Cela les oblige, en cas de dégâts causés par une catastrophe naturelle comme une tempête ou une inondation, à puiser dans leur patrimoine propre. Jouissant de moins d'actifs, il est plus difficile pour eux de faire face au risque. Leurs actifs sont aussi moins diversifiés: pour les ménages pauvres urbains, le logement constitue l'essentiel de leur patrimoine (Moser, 2007) et est à risque en cas d'événement extrême. Pour les ménages modestes ruraux, ce sont les troupeaux qui représentent l'essentiel de leur capital, susceptibles d'être perdus en cas de sécheresse (Nkedianye et al., 2011). En cas d'aléas climatiques, les plus modestes sont également plus touchés par les maladies comme la malaria, ou les maladies d'origine hydrique (Hallegatte et al., 2015). Un choc environnemental se traduit par des effets sur le long-terme pour les plus modestes, augmentant leurs chances de tomber dans des trappes de pauvreté (Carter et al., 2007). Ainsi, le changement climatique agit comme un amplificateur des risques pour les plus pauvres. 
Ces inégalités de vulnérabilités qui touchent les individus les plus désavantagés s'articulent avec d'autres phénomènes socio-économiques, tant à l'échelle des groupes sociaux qu'à celle des pays. La vulnérabilité est multidimensionnelle et peut être accentuée par différentes formes de discriminations dont sont victimes certains groupes, basées sur le genre, l'appartenance éthnique ou la classe sociale. Dans de nombreux pays en développement, ce sont les femmes qui sont chargées de la collecte de l'eau et du bois de chauffe, ce qui les rend vulnérables aux effets du réchauffement climatique (Egeru, Kateregga et Majaliwa, 2014) (GIEC, Cinquième rapport d'évaluation, Groupe 2, Chapitre 13). Loin de se limiter à la seule dimension des revenus, la race, la structure familiale, ou le niveau d'éducation peuvent jouer un rôle dans la façon dont les individus sont affectés par des catastrophes naturelles, comme dans le cas de l'Ouragan Katrina (Elliott et Pais, 2006 ; Logan, 2006 ; Masozera, Bailey et Kerchner, 2007 ; Myers, Slack et Singelmann, 2008). Cette situation est renforcée par le fait que les groupes défavorisés ont souvent moins de pouvoir décisionnaire et bénéficient moins des ressources publiques.

Le changement climatique risque donc d'accentuer les inégalités existantes. On peut déjà mesurer un effet du changement climatique plus important pour les plus pauvres, et ce à toutes les échelles. Le changement climatique a eu tendance à accentuer les inégalités entre pays, et une étude suggère que le ratio entre dernier et premier déciles serait $25 \%$ moins élevé s'il n'y avait pas eu de changement climatique (Diffenbaugh et Burke, 2019). L'impact du changement climatique porte de manière disproportionnée sur les plus défavorisés au sein des pays entre différentes régions et à l'intérieur des villes. Sans action pour limiter le changement climatique, ses impacts continueraient à avoir un effet amplificateur des inégalités - entre pays et au sein de pays - et pourraient compromettre le développement et l'éradication de la pauvreté (King et Harrington, 2018; Bathiany et al., 2018; Hallegatte et Rozenberg, 2017). Un rapport de la Banque mondiale estime que 100 millions de personnes supplémentaires pourraient basculer dans la pauvreté en 2030 du fait du changement climatique (Hallegatte et al., 2015). La maîtrise du réchauffement climatique est donc une des conditions de l'amélioration durable des niveaux de vie. 


\section{Les pays et les individus riches contribuent de façon disproportionnée au changement climatique}

Si les pays et les individus les plus pauvres sont les plus vulnérables aux impacts du changement climatique, ce sont à l'inverse les plus riches qui sont majoritairement responsables des émissions de gaz à effet serre dont l'accumulation dans l'atmosphère cause le changement climatique.

Alors que certains pays émergents ont commencé à dépasser les pays développés en termes d'émissions totales actuelles - la Chine, est aujourd'hui de loin le plus grand émetteur de dioxyde de carbone (Quéré et al., 2018) - il subsiste une disparité entre pays développés et pays en développement en termes d'émissions par habitant et d'émissions historiques totales, et donc de contributions au réchauffement planétaire observé. Les émissions de gaz à effet de serre territoriales restent aujourd'hui principalement liées au niveau de richesse et de développement des pays : rapportées à la population, les émissions des États-Unis atteignent près de $20 \mathrm{tCO}$-eq/personne/an, celles de I'Union européenne et de la Chine sont proches de 8 tCO2-eq/ personne/an, celles de I'Inde à peine plus de 2 tCO2-eq/personne/an et celles du Sénégal ou du Burkina Fasso par exemple se situent entre 1 et 2 tCO2-eq/personne/an (Ritchie et Roser, 2017).

Si les émissions dues à la production de biens sont réattribuées aux pays où les biens sont consommés, l'écart entre pays développés et en développement se creuse davantage par rapport à l'écart en émissions territoriales (Peters et al., 2011 ; Karstensen, Peters et Andrew, 2013 ; Caro et al., 2014). Les pays développés sont en effet globalement importateurs d'émissions « incorporées » dans le commerce et les pays émergents et en développement en sont exportateurs.

Enfin, si l'on cherche à attribuer aux différents pays la responsabilité historique du forçage radiatif additionnel ou du réchauffement planétaire observé aujourd'hui (Carte 3), la contribution des pays développés est plus importante que leur part des émissions actuelles car, ayant été les premiers à engager la révolution industrielle, ils ont participé à l'accumulation de gaz à effet de serre dans l'atmosphère depuis plus longtemps. Selon le choix de l'année à partir de laquelle commencer à comptabiliser les émissions, l'inclusion ou l'exclusion des émissions dues au changement d'affectation des terres (la déforestation notamment) et des gaz autres que le $\mathrm{CO} 2$, les contributions relatives changent significativement (Höhne et al., 2011 ; Den Elzen et al., 2013 ; Matthews 
et al., 2014 ; Matthews, 2016). Néanmoins, il ressort que la responsabilité historique du réchauffement observé est majoritairement portée par les pays industrialisés (qui représentent plus de $55 \%$ des émissions cumulées depuis 1850), mais aussi par les pays qui connaissent des niveaux élevés de déforestation. La part de la responsabilité historique imputable aux pays émergents et en développement est cependant logiquement en augmentation à mesure que le temps passe, notamment celles de la Chine et de l'Inde, et elle pourrait dépasser celle des pays développés vers 2030 (Ward et Mahowald, 2014).

Carte 3. Émissions cumulées par habitant
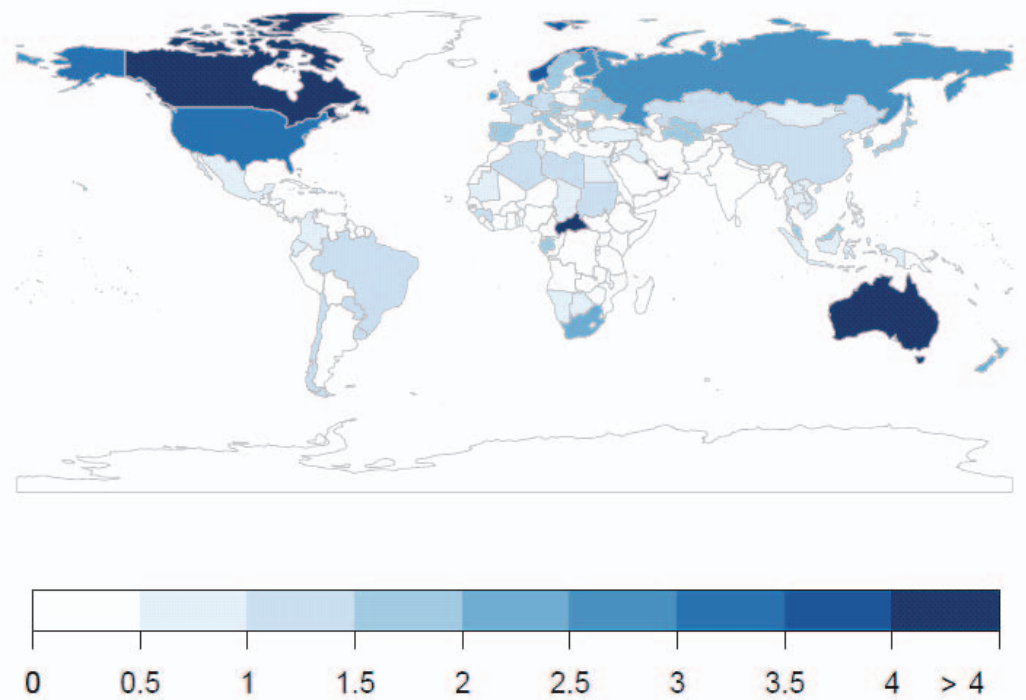

Note : Émissions cumulées par habitant normalisées pour les 130 pays dont la population dépasse un million d’habitants. Cet indicateur permet de quantifier la responsabilité des pays.

Source : Données de Frame et al., 2019.

Au sein des pays, il existe également de fortes disparités dans l'empreinte carbone des ménages. Si le niveau de richesse d'un individu n'est pas le seul déterminant de ses émissions (les autres déterminants étant sa localisation urbaine/rurale, son âge, etc.), il en reste le premier. Cela a en particulier été montré pour les ménages européens (Ivanova et al., 2017 ; Sommer et Kratena, 2017), américains (Jorgenson, Schor et Huang, 2017) et chinois (Wiedenhofer et al., 2017 ; Chen et al., 2019). En France, l'empreinte carbone d'un ménage du décile de revenu le plus élevé est presque trois fois 
supérieure à celle d'un ménage du décile le moins élevé (voir graphique 1). En analysant l'indice de Palma « carbone » - i.e. le ratio des émissions des $10 \%$ des individus les plus émetteurs par rapport à celles des $40 \%$ les moins émetteurs - il apparaît que ce ratio est plus élevé dans les pays en développement que dans les pays développés (Pan et al., 2019). À l'échelle mondiale, l'indice de Palma «carbone » est plus élevé qu'à l'intérieur de n'importe quel pays, ce qui reflète une inégalité très marquée lorsque l'on considère les émissions individuelles au-delà des limites territoriales. Le développement rapide de la Chine et des autres pays émergents a réduit les inégalités d'émissions entre pays ces dernières décennies, mais ce mouvement s'est accompagné d'une augmentation dans les inégalités d'émissions au sein des pays. Ainsi, aujourd'hui, à l'échelle mondiale, les $10 \%$ des ménages les plus émetteurs sont responsables d'environ $40 \%$ des émissions de gaz à effet de serre, tandis que les $40 \%$ les moins émetteurs représentent moins de $8 \%$ des émissions (Piketty et Chancel, 2015).

De plus, toutes les émissions ne peuvent être mise sur un même plan d'un point de vue éthique. Parmi les émissions, il convient en effet de distinguer celles qui sont de l'ordre de la subsistance de celles qui constituent un «luxe»(Shue, 1993; 2019). Par exemple, peut-on

\section{Graphique 1. Empreinte carbone des ménages français par décile de revenu} En tCO2e

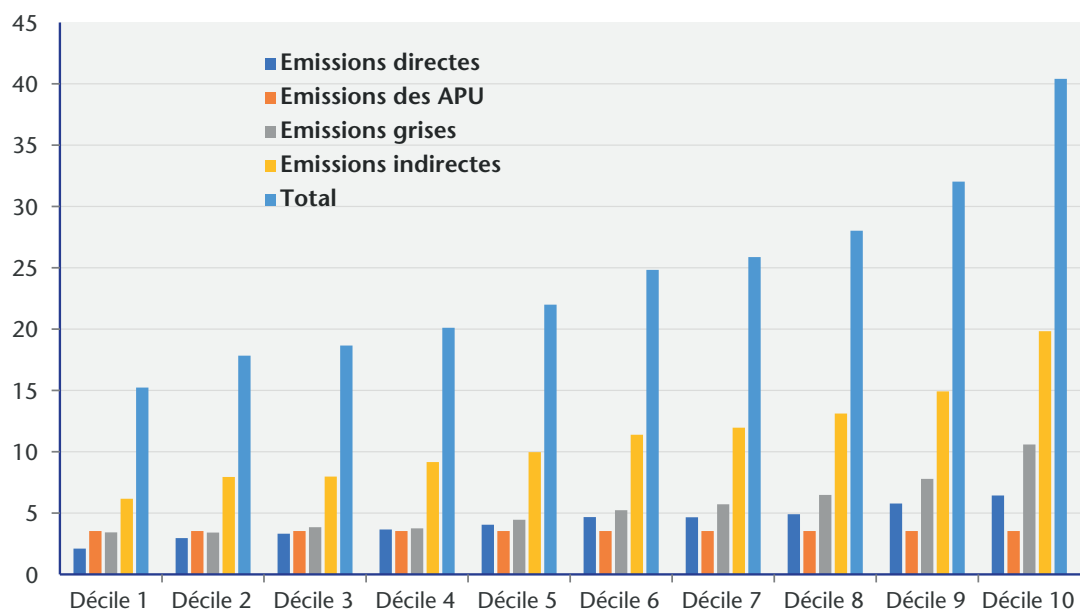

Note: L'empreinte se décompose en émissions directes (émises au moment de la consommation), émissions indirectes (émises lors de la production du bien ou service consommé), émissions grises (induites par l'ensemble des activités en amont de la chaîne de valeur), et émissions des Administrations Publiques (APU). Les empreintes sont calculées par ménage pour prendre en compte les effets de composition au sein du foyer. Lorsque l'on raisonne par individu, l'écart entre les déciles est légèrement réduit.

Source : ADEME, Malliet et al., 2019, «La fiscalité carbone aux frontières ». 
considérer qu'une tonne de $\mathrm{CO} 2$ émise pour se déplacer vers un lieu de vacances lointaines et une tonne de $\mathrm{CO} 2$ émise pour la production d'une denrée alimentaire de base sont à mettre sur le même plan ? Ainsi, il s'agit de traduire le principe de l'équité, présent dans les textes des négociations internationales sur le climat depuis la Convention Cadre des Nations Unies sur le Changement Climatique de 1992 et repris dans I'Accord de Paris qui souligne que « l'action et la riposte face aux changements climatiques et les effets des changements climatiques sont intrinsèquement liés à un accès équitable au développement durable et à l'élimination de la pauvreté ».

En s'appuyant sur l'approche par les capacités et les besoins fondamentaux, certains auteurs (Rao et Baer, 2012; Rao et Min, 2018b ; O'Neill et al., 2018) ont interprété ce principe d'accès équitable au développement durable en définissant un ensemble de conditions matérielles universelles, irréductibles et essentielles pour atteindre le bien-être humain de base, ainsi que des indicateurs et des seuils quantitatifs associés. Ils définissent ainsi un niveau de « vie décente » (Rao et Baer, 2012 ; Rao et Min, 2018b) ou un espace de développement « sûr et juste » (O'Neill et al., 2018), à travers des indicateurs mesurant la satisfaction des besoins humains fondamentaux (nutrition adéquate, logement, accès à des soins de santé, à l'éducation, etc.). Ils quantifient ensuite les besoins énergétiques et les émissions associées à ces indicateurs. Il se dégage un consensus sur le fait que la sortie de l'extrême pauvreté ou l'accès universel à l'énergie peuvent être atteints sans représenter des émissions des gaz à effet de serre importantes (Tait et Winkler, 2012 ; Pachauri, 2014 ; Chakravarty et Tavoni, 2013 ; Rao, Riahi et Grubler, 2014 ; Pachauri et al., 2013).

Pour autant, les études donnent des résultats divergents sur le sens de l'effet d'une réduction des inégalités sur les émissions, conduisant à une augmentation ou une diminution des émissions (Hubacek, Baiocchi, Feng et Patwardhan, 2017 ; Grunewald et al., 2017 ; Rao et Min, 2018a). L'effet absolu reste toutefois modéré : Rao et Min (2018b) bornent par exemple à $8 \%$ l'augmentation maximale plausible des émissions qui accompagnerait la réduction du coefficient de Gini mondial de son niveau actuel de 0.55 à un niveau de 0.3 .

Enfin, plusieurs études concluent que l'atteinte de niveaux de revenu plus importants que l'extrême pauvreté et l'atteinte d'objectifs sociaux plus qualitatifs sont associés à des émissions plus élevées (Hubacek, Baiocchi, Feng, Castillo et al., 2017 ; Scherer et al., 2018 ; 
O'Neill et al., 2018). Cela appelle des politiques qui sont à même de prendre en compte à la fois les objectifs d'atténuation et ceux de réduction des inégalités, notamment en portant sur l'intensité carbone des modes de vie (Scherer et al., 2018), I'attention à la sobriété et à l'équité (O'Neill et al., 2018) et ciblant les personnes à l'autre extrémité de l'échelle sociale - les super-riches (Otto et al., 2019).

\section{Effets distributifs et équité de l'action face au changement climatique}

Compte-tenu des liens qui viennent d'être détaillés entre changement climatique et inégalités, il est essentiel d'articuler les politiques de réduction des émissions de gaz à effet de serre à leurs effets sur les inégalités présentes et futurs. La prise en compte des effets distributifs de l'atténuation, tant en termes de répartition des bénéfices dus aux impacts du changement climatique évités que de répartition des coûts de l'atténuation, permet d'éclairer le niveau d'ambition des politiques climatiques et le juste partage des actions d'atténuation, et de leur financement, entre les différents pays. Les politiques d'atténuation et d'adaptation peuvent en effet avoir des effets régressifs ou progressifs, augmenter ou diminuer les inégalités et la pauvreté, selon la façon dont elles sont conçues et mises en œuvre.

Les inégalités de répartition des dommages climatiques à venir justifient des politiques d'atténuation plus ambitieuses. Réduire les émissions aujourd'hui limite les risques futurs pour les plus vulnérables de faire face à des événements extrêmes ou des impacts sur leur santé. La réduction des inégalités futures peut ainsi être vu comme un «cobénéfice » de l'atténuation. Ce bénéfice peut se mesurer à l'aide d'un outil d'analyse économique appelé valeur sociale du carbone, qui correspond à la valeur actualisée des dommages évités, mais aussi à la valeur à donner aux actions d'atténuation. Cette valeur est notamment utilisée pour faire l'analyse coûts-bénéfices de politiques publiques, de projets d'investissement publics ou pour dimensionner une fiscalité carbone. Déterminer cette valeur soulève des questions philosophiques et éthiques sur la prise en compte du risque et la valorisation des inégalités (Fleurbaey et al,. 2019), mais le fait que les impacts pèsent plus fortement sur les classes de revenus les plus faibles leur donne un poids plus important. Cela peut augmenter la valeur de l'atténuation d'un facteur compris entre 2 et 10 (Dennig et al., 2015 ; Anthoff et Emmerling, 2018) (graphique 2). L'ampleur de cet effet 
peut être limitée lorsque les coûts de l'atténuation pèsent de manière disproportionnée sur les plus vulnérables (Budolfson et al., 2017). Néanmoins, même lorsque les coûts sont partagés de manière régressive entre pays, l'atténuation permet de réduire les inégalités à longterme dans de nombreux scénarios socioéconomiques futures (Taconet et al., 2020).

Graphique 2. Valeur sociale du carbone en 2005

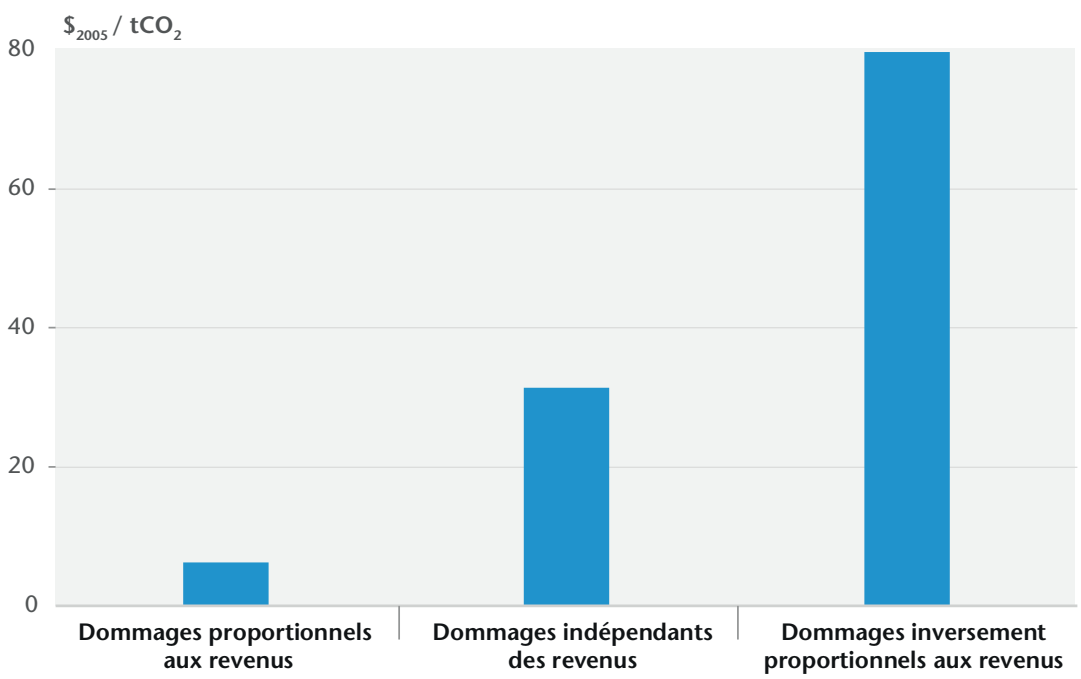

Note: Valeur sociale du carbone en 2005, selon I'hypothèse faite sur la relation entre répartition des dommages du changement climatique et la répartition des revenus : des dommages proportionnels aux revenus, indépendants des revenus ou inversement proportionnels aux revenus.

Source : Dennig et al., 2015.

Définir la juste répartition des actions d'atténuation, et de leur financement entre pays s'avère délicat, tant par la difficile prise en compte des différents niveaux d'interactions entre inégalités et climat que compte tenu des différentes visions du juste (Pottier et al., 2017). Dans les négociations sur le climat, les pays ont cherché à définir au cours des différentes COP la répartition équitable des réductions d'émissions entre pays et des obligations de financements et de transferts internationaux, respectueux à la fois de la responsabilité historique des pays et de leurs capacités différentes. Cela a notamment abouti à l'adoption du principe de «responsabilité commune mais différenciée » dans la Convention-cadre des Nations Unies sur les changements climatiques (CCNUCC) puis au sein du Protocole de Kyoto dans les années 1990. Mais en y regardant de plus près, de nombreuses 
questions se posent pour rendre ce concept opérationnel. Faut-il compenser les pays qui seront plus affectés par le changement climatique (De Cian et al., 2016) ? Comment prendre en compte le besoin de développement tout en limitant l'augmentation de la température à $2^{\circ} \mathrm{C}$ (Winkler, Letete et Marquard, 2013) ? Comment attribuer la responsabilité des émissions entre la production et le lieu de consommation? Faut-il donner une priorité aux plus pauvres et comment créer des exemptions pour des émissions correspondant aux besoins de base des populations les plus pauvres (Rao, 2014; Chakravarty et al., 2009) ? Faut-il mettre sur le même plan les inégalités du fait de l'externalité carbone de celles existant en dehors de la question climatique (Gosseries, 2005) ?

La reconnaissance de la responsabilité historique des pays développés a amené les rédacteurs du Protocole de Kyoto à imposer des réductions d'émissions uniquement aux pays dits de l'Annexe 1, et à proposer des mécanismes de financement Nord-Sud, notamment le Mécanisme de Développement propre et de transferts de technologies. Le Protocole de Kyoto devait être une première étape vers un accord universel de réduction des émissions qui devait rentrer en vigueur après 2012. L'approche top-down consistant à se partager le fardeau de l'effort de réduction des émissions a été abandonnée après la COP de Copenhague (2009) du fait de l'impossibilité à définir un partage équitable aux vues de tous. Dans le cadre de l'Accord de Paris, c'est à chaque pays de définir sa contribution aux réductions d'émissions à travers les Nationally Determined Contributions (NDC). Les NDC, si exactement atteintes, contribueraient à l'horizon 2030 à une réduction des inégalités d'émissions par habitant entre pays, avec une réduction pour les principaux pays de I'OCDE et une augmentation pour les pays émergents et en développement (Benveniste et al., 2018) (graphique 3). Néanmoins les émissions résultantes en 2030 seraient trop élevées pour être compatibles avec l'objectif de l'Accord de Paris de contenir l'augmentation de la température moyenne mondiale bien en-deçà de $+2^{\circ} \mathrm{C}$ par rapport à l'ère préindustrielle. Par rapport à un scénario de réduction des émissions plus ambitieux à court-terme, les NDC sont défavorables en termes d'équité intergénérationnelle, mais aussi intra-générationnelles future car les générations futures auraient à supporter le coût de réductions d'émissions très rapides après 2030 et/ou des impacts plus importants du changement climatique, impacts qui sont inégalement répartis (Liu, Fujimori et Masui, 2016). Dans la perspective de la révision des NDC, devant conduire à une ambition 
accrue, plusieurs études (Robiou du Pont et al., 2017 ; Kartha et al., 2018 ; van den Berg et al., 2019) ont évalué les NDC actuelles à l'aune des principaux critères de partage du fardeau de l'atténuation proposés (convergence des émissions par habitant, égalité des émissions cumulées par habitant, capacité à payer, ...). Selon le critère les émissions qui seraient allouées à un pays donné varient beaucoup et certains critères conduisent à des budgets d'émissions négatifs pour les pays développés (voir par exemple http://www.ccalc.ethz.ch ou parisequity-check.org).

Si la question de l'équité et de ce que serait une ambition juste pour les NDC des différents pays continuera de jouer un rôle dans les négociations internationales, l'objectif de long-terme de l'Accord nécessite que chaque pays tende vers la neutralité carbone, à un rythme dépendant de ses conditions spécifiques et la question de l'équité se déplace davantage vers la question du financement des actions (Holz, Kartha et Athanasiou, 2018).

\section{Graphique 3. Évolution des émissions de gaz à effet de serre par habitant}

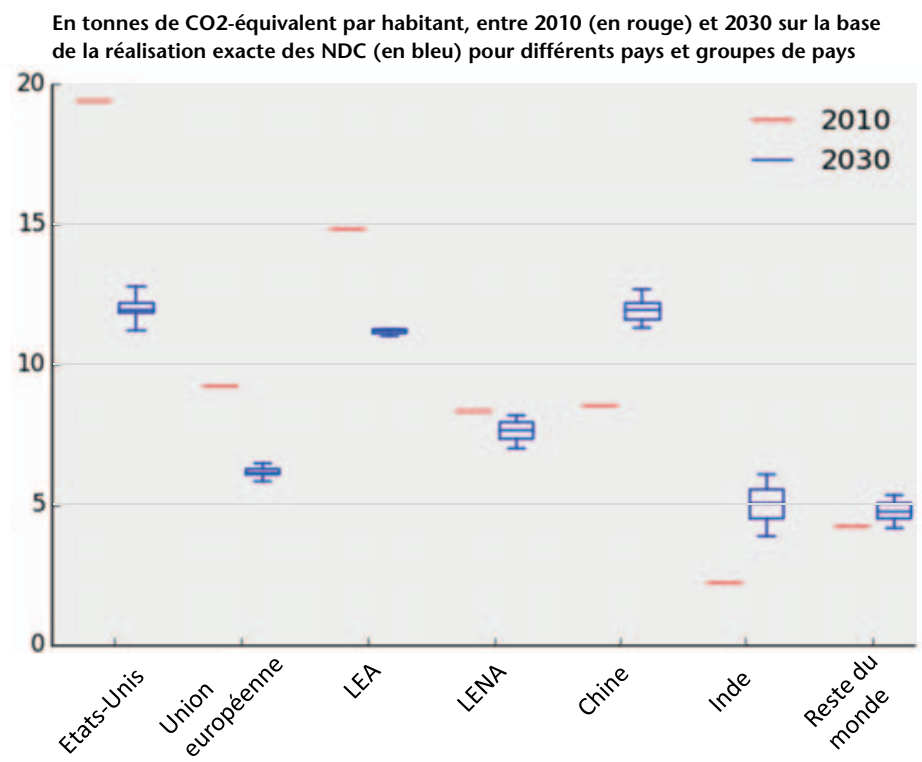

Note : Les fourchettes d'incertitude pour 2030 sont présentées avec les valeurs du 5e percentile, du 1er quartile, de la médiane, du 3e quartile et du 95e percentile. LEA signifie Large Emitters avec NDCs contenant une réduction Absolue par rapport à une année de référence (regroupant l'Australie, le Brésil, le Canada, le Japon, le Kazakhstan, la Russie et l'Ukraine). LENA est l'abréviation de Large Emitters avec NDCs Ne contenant pas une réduction Absolue par rapport à une année de référence (regroupant l'Egypte, I'Indonésie, I'Iran, la Corée du Sud, la Malaisie, le Mexique, I'Arabie Saoudite, I'Afrique du Sud, Taiwan, la Thailande, la Turquie et les Emirats Arabes Unis).

Source : Materiel supplémentaire à Benveniste et al., 2018. 
Enfin les actions pour réduire les émissions de gaz à effet de serre et s'adapter à un climat qui change ne doivent pas négliger leur propre effet sur les inégalités et sur la pauvreté et la précarité. Les politiques climatiques induisent des coûts et des bénéfices pour différents individus au sein d'un pays. Ces politiques peuvent être régressives, c'est-à-dire représenter un coût plus important pour les plus modestes en proportion de leurs revenus (Bento, 2013). En effet, ces politiques risquent d'augmenter les prix des biens intensifs en émissions qui représentent une part plus grande des dépenses des plus pauvres. Le passage à des technologies plus propres, qui sont parfois plus intensives en capital, joue également sur les revenus. Ces effets dépendent à la fois du type d'instrument de politique mobilisé et de la façon dont ils sont mis en place.

Par exemple la fiscalité sur les émissions induit d'importants effets distributifs. Ces effets sont plus importants dans certains secteurs comme le transport et dans les pays développés que dans les pays en développement, dans lesquels la consommation d'énergie des ménages modestes est faible (Dorband et al., 2019 ; Ohlendorf et al., 2018). L'impact des fiscalités sur le carbone dépend également des effets sur les revenus du travail et du capital (Goulder et al., 2019), de la façon dont les consommateurs ajustent leur comportement face à des variations de prix ainsi que des variations de revenus au cours de leur vie (Ohlendorf et al., 2018). En France, une taxe carbone sur les secteurs du transport et du logement a été instaurée en 2014 et son niveau doit progresser chaque année avec un risque pour les ménages dépendant de la voiture ou résidant dans des logements mal isolés. L'effet d'une taxe à 30 euros/tCO2 (son niveau de 2017) augmente ainsi le nombre de personnes en situation de précarité énergétique d'environ $6 \%$ (Berry, 2019). Mais l'instauration d'une taxe s'accompagne de revenus fiscaux additionnels dont I'usage conditionne son équité (graphique 4). L'augmentation de la précarité énergétique induite par la taxe carbone peut être compensée en redistribuant une partie des revenus aux ménages : il suffit d'utiliser $15 \%$ des recettes pour annuler l'effet sur la précarité énergétique (Berry, 2019). Bien que les $10 \%$ les plus modestes puissent en moyenne bénéficier de la redistribution, il subsiste néanmoins une proportion importante de ménages qui voit sa situation se dégrader du fait d'une grande hétérogénéité à l'intérieur des déciles (Douenne, s. d.). Il en va de même pour les permis d'émissions, les effets distributifs dépendent des règles d'attribution, l'allocation gratuite favorisant les propriétaires des 
entreprises polluantes (Dinan et Rogers, 2002 ; Parry, 2004). Enfin les réformes visant à supprimer les subventions aux énergies fossiles peuvent être bénéfiques lorsqu'elles sont remplacées par des transferts directs (Durand-Lasserve et al., 2015 ; Vogt-Schilb et al., 2019).

Graphique 4. Part du revenu des ménages consacrée à la fiscalité carbone par décile

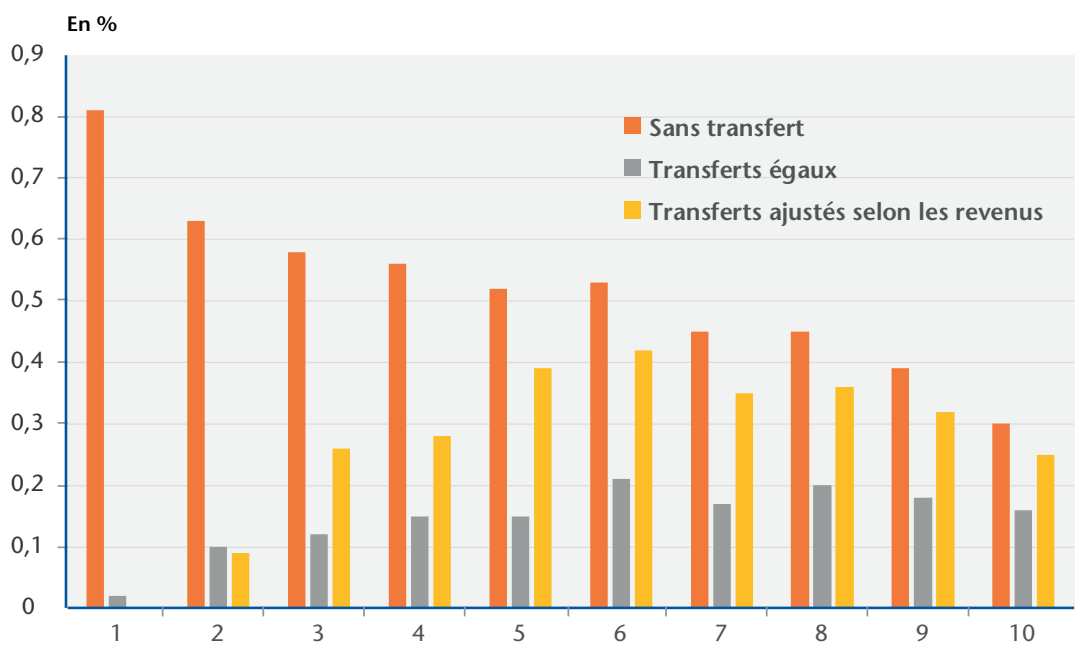

Note : Part moyenne des revenus des ménages français, par décile, consacrée à la fiscalité carbone (sans transfert) et part restante après reversement d'une partie des revenus de la fiscalité aux ménages, selon deux modalités visant à compenser la régressivité de la taxe (selon l'indice de Suits) : I'une avec des montants de transferts égaux pour tous les ménages (dans ce cas, compenser la régressivité implique de redistribuer $59 \%$ des revenus), la seconde avec des montants de transferts ajustés selon les revenus de façon inversement proportionnelle (dans ce cas, compenser la régressivité implique de redistribuer $33 \%$ des revenus).

Source : Berry, 2019.

D'autres politiques publiques visant à réduire les émissions peuvent également avoir un effet négatif sur les plus modestes. Les normes imposant un standard d'efficacité énergétique sur les moteurs, si elles permettent des économies d'émissions, renchérissent également le coût d'acquisition des véhicules pour les plus modestes (Levinson, 2016). Pour atteindre la même réduction d'émissions, les normes peuvent être plus régressives que les taxes (Fullerton, 2017). De même, les standards d'efficacité dans la construction de bâtiments en Californie ont eu un effet négatif sur les plus modestes et se sont traduits par une réduction de la surface de leurs logements (Bruegge, Deryugina et Myers, 2019). L'effet distributif des subventions aux énergies renouvelables varie selon leur design, en particulier la façon dont sont établis les prix sur le marché de l'électricité et la capacité des producteurs à transférer les coûts aux consommateurs (Reguant, 2018). Enfin, 
les crédits d'impôts pour l'installation de panneaux solaires ou l'achat de véhicules électriques peuvent être captés par les plus riches. Aux États-Unis, 60 \% des différents crédits d'impôts « verts » entre 2006 et 2013 ont bénéficié aux $20 \%$ les plus riches (Borenstein et Davis, 2016). En France, l'ensemble des mesures existantes pour réduire la facture énergétique des ménages (crédits d'impôts ou prêt pour la rénovation, subventions, chèque énergie, ...) sont insuffisantes pour attendre les objectifs de réduction de la précarité énergétique et sont coûteuses, si bien que des transferts monétaires directs pourraient être plus efficaces pour combattre la précarité énergétique (Charlier, Risch et Salmon, 2018).

Enfin, certaines politiques d'atténuation affectent les plus pauvres via des effets sur les prix de l'alimentation. Ainsi, le développement du biocarburant peut avoir un effet délétère sur la sécurité alimentaire (Hasegawa et al., 2018 ; Fugimori et al., 2019). L'utilisation de terres pour la production de biocarburants renchérit le prix des denrées alimentaires et pourrait avoir des impacts négatifs, particulièrement dans les régions à faible revenu comme l'Afrique subsaharienne et I'Asie du Sud. La mise en culture de certaines terres pour produire du biocarburant pourrait également entraîner de la déforestation et déposséder certaines communautés de leurs terres.

Inversement, certaines politiques d'atténuation présentent des cobénéfices pour les plus vulnérables. En effet la combustion d'énergies fossiles s'accompagne d'émissions de polluants locaux comme les particules fines, ou les oxydes d'azote qui causent des maladies cardiorespiratoires (Smith et al., 2013). Ce sont les communautés les plus désavantagées socialement qui sont les plus exposées à ces risques sur la santé l'échelle globale (Hajat, Hsia et O'Neill, 2015). Elles pourraient donc bénéficier de la réduction de l'utilisation de véhicules à moteurs thermiques ou de la limitation du recours au charbon. De même, I'utilisation de fourneaux plus efficaces permet de limiter les émissions de gaz à effet de serre tout en améliorant la qualité de l'air et donc la santé des utilisateurs (Rao et al., 2013).

Les politiques d'adaptation soulèvent des enjeux analogues et peuvent avoir des effets importants sur les ménages modestes. Certaines actions d'adaptation permettent de diminuer la vulnérabilité des plus pauvres aux aléas climatiques, comme la conversion à des cultures agricoles plus résistantes. Le développement des services financiers destinés aux plus modestes, qui en sont souvent exclus, 
améliore leur capacité à faire face à des imprévus notamment climatiques. L'indexation des transferts monétaires sur les prix alimentaires pourrait également accompagner les ménages en cas de pic des prix de I'alimentation (Shock Wave, 2015). Cependant, à l'heure actuelle, les dépenses d'adaptation semblent se porter davantage sur la protection du capital physique plutôt que sur celle des populations à risque (Georgeson et al., 2016). À ce titre, certains outils de la décision publique tels que l'analyse coût-bénéfice, qui ne tiennent pas compte de la distribution des bénéfices futurs, favorisent les projets qui ont les bénéfices monétaires les plus élevés au détriment de ceux permettant de mieux protéger les plus vulnérables. La prise en compte des effets sur le bien-être, et non seulement les bénéfices monétaires absolus, changerait les arbitrages.

\section{Conclusion}

Le changement climatique agit comme un amplificateur d'inégalités en touchant les plus défavorisés à toutes les échelles, qui sont plus vulnérables aux impacts du changement climatique. La prise en compte de ces inégalités d'impact donne plus de valeur aux actions d'atténuation des émissions de gaz à effet de serre et doit conduire à des politiques plus ambitieuses en la matière.

Dans la mesure où les niveaux d'émissions diffèrent entre pays et entre individus, où les coûts de la réduction des émissions et les bénéfices des impacts évités sont inégalement répartis entre les individus et entre les pays, les questions d'équité au sein de chaque génération sont essentielles pour définir des trajectoires bas carbone justes, respectueuses des besoins des générations présentes et des intérêts des générations futures (Klinsky et al., 2017 ; Klinsky et Winkler, 2018).

Les politiques de réductions d'émissions peuvent en outre avoir des impacts pour les plus modestes. À l'échelle internationale, il s'agit de réduire les émissions sans entraver l'accès au développement, notamment des pays les moins avancés, et ainsi accompagner l'éradication de la pauvreté. À l'intérieur d'un pays, la réduction des émissions pose la question de la justice de la transition. Selon le type de politiques publiques qui sont mises en place, les plus modestes peuvent être affectés de manière disproportionnée. 
Les études sur le sujet montrent que climat et égalité ne s'opposent pas nécessairement et qu'il existe des moyens d'articuler politiques climatiques et justice sociale. Mais cela suppose d'abord de reconnaître les contradictions potentielles entre justice sociale et politiques climatiques et ensuite de mettre en place des dispositifs d'accompagnement et de compensation.

\section{Références}

ADEME, Paul Malliet, Ruben Haalebos et Emeric Nicolas, 2019, La fiscalité carbone aux frontières : ses impacts redistributifs sur le revenu des ménages français.

Ahmadalipour Ali, Hamid Moradkhani et Mukesh Kumar, 2019, « Mortality risk from heat stress expected to hit poorest nations the hardest ", Climatic Change, vol. 152, n 3-4, pp. 569-79.

Althor Glenn, James E. M. Watson et Richard A. Fuller, 2016, "Global Mismatch between Greenhouse Gas Emissions and the Burden of Climate Change», Scientific Reports 6 (février), n²0281. https:// doi.org/10.1038/srep20281.

Alvaredo Facundo, Lucas Chancel, Thomas Piketty, Emmanuel Saez et Gabriel Zucman, 2018, «World Inequality Report 2018 ». http:// wir2018.wid.world/.

Anthoff David et Johannes Emmerling, 2018, « Inequality and the Social Cost of Carbon », Journal of the Association of Environmental and Resource Economists, vol. 6, n 2, pp. 243-73. https://doi.org/10.1086/701900.

Bathiany Sebastian, Vasilis Dakos, Marten Scheffer et Timothy M. Lenton, 2018, "Climate Models Predict Increasing Temperature Variability in Poor Countries», Science Advances, vol. 4, n 5. eaar5809. https:// doi.org/10.1126/sciadv.aar5809.

Benveniste Hélène, Olivier Boucher, Céline Guivarch, Hervé Le Treut et Patrick Criqui, 2018, "Impacts of Nationally Determined Contributions on 2030 Global Greenhouse Gas Emissions: Uncertainty Analysis and Distribution of Emissions », Environmental Research Letters, vol. 13 $\mathrm{n}^{\circ}$ 1. 014022. https://doi.org/10.1088/1748-9326/aaa0b9.

Berg Nicole J. van den, Heleen L. van Soest, Andries F. Hof, Michel G. J. den Elzen, Detlef P. van Vuuren, Wenying Chen, Laurent Drouet et al., 2019, « Implications of Various Effort-Sharing Approaches for National Carbon Budgets and Emission Pathways ", Climatic Change, février. https://doi.org/10.1007/s10584-019-02368-y.

Berry Audrey, 2019, « The Distributional Effects of a Carbon Tax and Its Impact on Fuel Poverty: A Microsimulation Study in the French 
Context », Energy Policy, n 124, janvier, pp. 81-94. https://doi.org/ 10.1016/j.enpol.2018.09.021.

Borenstein Severin et Lucas W. Davis, 2016, « The Distributional Effects of US Clean Energy Tax Credits », Tax Policy and the Economy, vol. 30, $\mathrm{n}^{\circ}$ 1, pp. 191-234. https://doi.org/10.1086/685597.

Borrell Carme, Marc Marí-Dell'Olmo, Maica Rodríguez-Sanz, Patrícia Garcia-Olalla, Joan A. Caylà, Joan Benach et Carles Muntaner, 2006, «Socioeconomic Position and Excess Mortality during the Heat Wave of 2003 in Barcelona », European Journal of Epidemiology, vol. 21, n ${ }^{\circ}$, pp. 633-40. https://doi.org/10.1007/s10654-006-9047-4.

Boustan Leah Platt, Matthew E. Kahn, Paul W. Rhode et Maria Lucia Yanguas, 2017, "The effect of natural disasters on economic activity in us counties: A century of data », National Bureau of Economic Research.

Braun Boris et Tibor Aßheuer, 2011, «Floods in megacity environments: vulnerability and coping strategies of slum dwellers in Dhaka/Bangladesh », Natural hazards, vol. 58, n², pp. 771-87.

Brouwer Roy, Sonia Akter, Luke Brander et Enamul Haque, 2007, « Socioeconomic Vulnerability and Adaptation to Environmental Risk: A Case Study of Climate Change and Flooding in Bangladesh », Risk Analysis, vol. 27, $\mathrm{n}^{\circ} 2$, pp. 313-26. https://doi.org/10.1111/j.1539-6924.2007. 00884.x.

Bruegge Chris, Tatyana Deryugina et Erica Myers, 2019, «The Distributional Effects of Building Energy Codes », Journal of the Association of Environmental and Resource Economists, vol. $6 \mathrm{n}^{\circ}$ S1, pp. 95-127.

Budolfson Mark, Francis Dennig, Marc Fleurbaey, Asher Siebert et Robert H. Socolow, 2017, « The Comparative Importance for Optimal Climate Policy of Discounting, Inequalities and Catastrophes ", Climatic Change, vol. 145, $\mathrm{n}^{\circ} 3-4$, pp. 481-94. https://doi.org/10.1007/s10584017-2094-X.

Byers Edward, Matthew Gidden, David Leclère, Juraj Balkovic, Peter Burek, Kristie Ebi, Peter Greve et al., 2018, "Global Exposure and Vulnerability to Multi-Sector Development and Climate Change Hotspots ", Environmental Research Letters, vol. 13, $\mathrm{n}^{\circ}$ 5, 055012. https://doi.org/ 10.1088/1748-9326/aabf45.

Caro Dario, Anna LoPresti, Steven J. Davis, Simone Bastianoni et Ken Caldeira, 2014, «CH4 and N2O emissions embodied in international trade of meat », Environmental Research Letters, vol. 9, n 11, 114005.

Carter Michael R., Peter D. Little, Tewodaj Mogues et Workneh Negatu, 2007, "Poverty Traps and Natural Disasters in Ethiopia and Honduras », World Development, vol. 35, $\mathrm{n}^{\circ}$ 5, pp. 835-56. https:// doi.org/10.1016/j.worlddev.2006.09.010.

Chakravarty Shoibal, Ananth Chikkatur, Heleen de Coninck, Stephen Pacala, Robert Socolow et Massimo Tavoni, 2009, "Sharing Global 
CO2 Emission Reductions among One Billion High Emitters », Proceedings of the National Academy of Sciences, vol. 106, $\mathrm{n}^{\circ} 29$, pp. 11884-88. https://doi.org/10.1073/pnas.0905232106.

Chakravarty Shoibal et Massimo Tavoni, 2013, «Energy poverty alleviation and climate change mitigation: Is there a trade off? », Energy economics, $\mathrm{n}^{\circ} 40$, pp. S67-73.

Charlier Dorothée, Anna Risch et Claire Salmon, 2018, «Energy Burden Alleviation and Greenhouse Gas Emissions Reduction: Can We Reach Two Objectives With One Policy?", Ecological Economics, n ${ }^{\circ} 143$, janvier, pp. 294-313. https://doi.org/10.1016/j.ecolecon.2017.07.002.

Chen Jiandong, Chong Xu, Lianbiao Cui, Shuo Huang et Malin Song, 2019, "Driving factors of CO2 emissions and inequality characteristics in China: A combined decomposition approach », Energy Economics, $\mathrm{n}^{\circ} 78$, pp. 589-97.

Crow Ben, Nichole Zlatunich et Brian Fulfrost, 2009, « Mapping Global Inequalities: Beyond Income Inequality to Multi-Dimensional Inequalities », Journal of International Development, vol. 21, $\mathrm{n}^{\circ}$ 8, pp. 1051-65. https://doi.org/10.1002/jid.1646.

De Cian E., A. F. Hof, G. Marangoni, M. Tavoni et D. P. van Vuuren, 2016, «Alleviating inequality in climate policy costs: an integrated perspective on mitigation, damage and adaptation », Environmental Research Letters, vol. 11, $\mathrm{n}^{\circ} 7,074015$.

Den Elzen Michel GJ, Jos GJ Olivier, Niklas Höhne et Greet Janssens-Maenhout, 2013, "Countries' contributions to climate change: effect of accounting for all greenhouse gases, recent trends, basic needs and technological progress », Climatic Change, vol. 121, n² 2, pp. 397-412.

Dennig Francis, Mark B. Budolfson, Marc Fleurbaey, Asher Siebert et Robert H. Socolow, 2015, «Inequality, Climate Impacts on the Future Poor, and Carbon Prices ", Proceedings of the National Academy of Sciences, vol. 112, $\mathrm{n}^{\circ}$ 52, pp. 15827-32. https://doi.org/10.1073/ pnas. 1513967112.

Deryugina Tatyana et Solomon M. Hsiang, 2014, « Does the environment still matter? Daily temperature and income in the United States », National Bureau of Economic Research.

Diffenbaugh Noah S. et Marshall Burke, 2019, "Global warming has increased global economic inequality », Proceedings of the National Academy of Sciences, vol. 116, $\mathrm{n}^{\circ} 20$, pp. 9808-13.

Dinan Terry M. et Diane Lim Rogers, 2002, «Distributional Effects of Carbon Allowance Trading: How Government Decisions Determine Winners and Losers ", National Tax Journal, vol. 55, n² 2, pp. 199-221.

Dorband Ira Irina, Michael Jakob, Matthias Kalkuhl et Jan Christoph Steckel, 2019, " Poverty and Distributional Effects of Carbon Pricing in Low- and Middle-Income Countries - A Global Comparative 
Analysis », World Development, $\mathrm{n}^{\circ} 115$, mars, pp. 246-57. https:// doi.org/10.1016/j.worlddev.2018.11.015.

Douenne Thomas, s. d., « The Vertical and Horizontal Distributive E?ects of Energy Taxes: A Case Study of a French Policy », n 43.

Durand-Lasserve Olivier, Lorenza Campagnolo, Jean Chateau et Rob Dellink, 2015, « Modelling of Distributional Impacts of Energy Subsidy Reforms: An Illustration with Indonesia », SSRN Scholarly Paper, ID 2633472, Rochester, NY, Social Science Research Network. https:// papers.ssrn.com/abstract=2633472.

Egeru Anthony, Eseza Kateregga et Gilber Jackson Mwanjalolo Majaliwa, 2014, "Coping with firewood scarcity in Soroti District of Eastern Uganda ", Open Journal of Forestry, vol. 4, n 1, p. 70.

Elliott James R. et Jeremy Pais, 2006, « Race, class, and Hurricane Katrina: Social differences in human responses to disaster ", Social science research, vol. 35, $\mathrm{n}^{\circ} 2$, pp. 295-321.

Emanuel Kerry, 2017, "Assessing the Present and Future Probability of Hurricane Harvey's Rainfall ", Proceedings of the National Academy of Sciences, vol. 114, $\mathrm{n}^{\circ} 48$, pp. 12681-84. https://doi.org/10.1073/pnas. 1716222114.

Firebaugh Glenn, 2015, "Global Income Inequality », In Emerging Trends in the Social and Behavioral Sciences, John Wiley \& Sons, Inc. https:// doi.org/10.1002/9781118900772.etrds0149.

Fleurbaey Marc, Maddalena Ferranna, Mark Budolfson, Francis Dennig, Kian Mintz-Woo, Robert Socolow, Dean Spears et Stéphane Zuber, 2019, « The Social Cost of Carbon: Valuing Inequality, Risk, and Population for Climate Policy», The Monist, vol. 102, $\mathrm{n}^{\circ}$ 1, pp. 84-109. https://doi.org/10.1093/monist/ony023.

Formetta Giuseppe, et Luc Feyen, 2019, « Empirical evidence of declining global vulnerability to climate-related hazards », Global Environmental Change, vol. 57 (juillet), 101920.

Frame David J., Luke J. Harrington, Jan S. Fuglestvedt, Richard J. Millar, Manoj M. Joshi et Simon Caney, 2019, « Emissions and Emergence: A New Index Comparing Relative Contributions to Climate Change with Relative Climatic Consequences", Environmental Research Letters, vol. 14, n 8, 084009. https://doi.org/10.1088/1748-9326/ab27fc.

Fullerton Don, 2017, Distributional effects of environmental and energy policy, Routledge.

Gosling Simon N. et Nigel W. Arnell, 2016, «A Global Assessment of the Impact of Climate Change on Water Scarcity», Climatic Change, vol. 134, n 3, pp. 371-85. https://doi.org/10.1007/s10584-013-0853-x.

Gosseries Axel, 2005, « Cosmopolitan Luck Egalitarianism and the Greenhouse Effect», Canadian Journal of Philosophy, $\mathrm{n}^{\circ} 35$ (sup1), pp. 279-309. https://doi.org/10.1080/00455091.2005.10716857. 
Goulder Lawrence H., Marc A. C. Hafstead, GyuRim Kim, et Xianling Long. 2019. "Impacts of a Carbon Tax across US Household Income Groups: What Are the Equity-Efficiency Trade-Offs? » Journal of Public Economics 175 (juillet) : 44-64. https://doi.org/10.1016/j.jpubeco. 2019.04.002.

Gregory Peter J. et Bruce Marshall, 2012, « Attribution of Climate Change: A Methodology to Estimate the Potential Contribution to Increases in Potato Yield in Scotland since 1960 », Global Change Biology, vol. 18, $\mathrm{n}^{\circ}$ 4, pp. 1372-88. https://doi.org/10.1111/j.1365-2486.2011.02601.x.

Grunewald Nicole, Stephan Klasen, Inmaculada Martínez-Zarzoso et Chris Muris, 2017, "The trade-off between income inequality and carbon dioxide emissions », Ecological Economics, n ${ }^{\circ}$ 142, pp. 249-56.

Hajat Anjum, Charlene Hsia et Marie S. O’Neill, 2015, « Socioeconomic Disparities and Air Pollution Exposure: A Global Review », Current Environmental Health Reports, vol. 2, $\mathrm{n}^{\circ} 4$, pp. 440-50. https://doi.org/ 10.1007/s40572-015-0069-5.

Hallegatte Stephane, Mook Bangalore, Marianne Fay, Tamaro Kane et Laura Bonzanigo, 2015, Shock waves: managing the impacts of climate change on poverty, World Bank Publications.

Hallegatte Stephane et Julie Rozenberg, 2017, "Climate change through a poverty lens », Nature Climate Change, vol. 7, n 4, pp. 250-56.

Harrington Luke J., David J. Frame, Erich M. Fischer, Ed Hawkins, Manoj Joshi et Chris D. Jones, 2016, " Poorest Countries Experience Earlier Anthropogenic Emergence of Daily Temperature Extremes », Environmental Research Letters, vol. 11, n 5, 055007. https://doi.org/10.1088/ 1748-9326/11/5/055007.

Heal Geoffrey et Jisung Park, 2016, « Reflections-Temperature Stress and the Direct Impact of Climate Change: A Review of an Emerging Literature ", Review of Environmental Economics and Policy, vol. 10, $\mathrm{n}^{\circ} 2$, pp. 347-62. https://doi.org/10.1093/reep/rew007.

Hertel Thomas W., 2015, "Food security under climate change », Nature Climate Change, vol. 6, $\mathrm{n}^{\circ} 1, \mathrm{p} .10$.

Höhne Niklas, Helcio Blum, Jan Fuglestvedt, Ragnhild Bieltvedt Skeie, Atsushi Kurosawa, Guoquan $\mathrm{Hu}$, Jason Lowe, Laila Gohar, Ben Matthews et Ana Claudia Nioac De Salles, 2011, "Contributions of individual countries' emissions to climate change and their uncertainty ", Climatic change, vol. 106, n 3, pp. 359-91.

Holz Christian, Sivan Kartha et Tom Athanasiou, 2018, "Fairly sharing 1.5: national fair shares of a 1.5 C-compliant global mitigation effort ", International Environmental Agreements: Politics, Law and Economics, vol. 18, $\mathrm{n}^{\circ} 1$, pp. 117-34.

Hubacek Klaus, Giovanni Baiocchi, Kuishuang Feng, Raúl Muñoz Castillo, Laixiang Sun et Jinjun Xue, 2017, " Global carbon inequality », Energy, Ecology and Environment, vol. 2, n 6, pp. 361-69. 
Hubacek Klaus, Giovanni Baiocchi, Kuishuang Feng et Anand Patwardhan, 2017, "Poverty eradication in a carbon constrained world», Nature communications, vol. 8, $\mathrm{n}^{\circ} 1$, p. 912 .

Huq Mainul, Malik Fida Khan, Kiran Pandey, Manjur Murshed Zahid Ahmed, Zahirul Huq Khan, Susmita Dasgupta et Nandan Mukherjee, 2010, Vulnerability of Bangladesh to cyclones in a changing climate: Potential damages and adaptation cost. The World Bank.

Ivanova Diana, Gibran Vita, Kjartan Steen-Olsen, Konstantin Stadler, Patricia C. Melo, Richard Wood et Edgar G. Hertwich, 2017, « Mapping the carbon footprint of EU regions ", Environmental Research Letters, vol. $12, \mathrm{n}^{\circ} 5,054013$.

Jaggard K. W., A. Qi et M. A. Semenov, 2007, « The Impact of Climate Change on Sugarbeet Yield in the UK: 1976-2004 », The Journal of Agricultural Science, vol. 145, $\mathrm{n}^{\circ}$ 4, pp. 367-75. https://doi.org/10.1017/ S0021859607006922.

Jongman Brenden, Hessel C. Winsemius, Jeroen C. J. H. Aerts, Érin Coughlan de Perez, Maarten K. van Aalst, Wolfgang Kron et Philip J. Ward, 2015, « Declining Vulnerability to River Floods and the Global Benefits of Adaptation ", Proceedings of the National Academy of Sciences, vol. 112, $\mathrm{n}^{\circ}$ 18, pp. E2271-80. https://doi.org/10.1073/pnas.1414439 112.

Jorgenson Andrew, Juliet Schor et Xiaorui Huang, 2017, « Income Inequality and Carbon Emissions in the United States: A State-Level Analysis, 1997-2012», Ecological Economics, vol. 134, avril, pp. 40-48. https:// doi.org/10.1016/j.ecolecon.2016.12.016.

Kahn Matthew E., 2005, « The death toll from natural disasters: the role of income, geography, and institutions ", Review of economics and statistics, vol. 87, n² 2, pp. 271-84.

Karstensen Jonas, Glen P. Peters et Robbie M. Andrew, 2013, «Attribution of $\mathrm{CO} 2$ emissions from Brazilian deforestation to consumers between 1990 and 2010 », Environmental Research Letters, vol. 8, n² 2, 024005.

Kartha Sivan, Tom Athanasiou, Simon Caney, Elizabeth Cripps, Kate Dooley, Navroz K. Dubash, Teng Fei et al., 2018, "Cascading Biases against Poorer Countries», Nature Climate Change, vol. 8, $\mathrm{n}^{\circ}$ 5, pp. 348-49. https://doi.org/10.1038/s41558-018-0152-7.

King Andrew D. et Luke J. Harrington, 2018, « The Inequality of Climate Change From 1.5 to $2^{\circ} \mathrm{C}$ of Global Warming », Geophysical Research Letters, vol. 45, $\mathrm{n}^{\circ}$ 10, pp. 5030-33. https://doi.org/10.1029/2018 GL078430.

Klinsky Sonja, Timmons Roberts, Saleemul Huq, Chukwumerije Okereke, Peter Newell, Peter Dauvergne, Karen O'Brien et al., 2017, «Why Equity Is Fundamental in Climate Change Policy Research ", Global Environmental Change, $\mathrm{n}^{\circ} 44$, mai, pp. 170-73. https://doi.org/10.1016/ j.gloenvcha.2016.08.002. 
Klinsky Sonja et Harald Winkler, 2018, «Building equity in: strategies for integrating equity into modelling for a $1.5 \mathrm{C}$ world», Philosophical Transactions of the Royal Society A: Mathematical, Physical and Engineering Sciences, vol. 376, $\mathrm{n}^{\circ} 2119,20160461$.

Laurent Éloi, 2011, « Issues in environmental justice within the European Union », Ecological Economics, vol. 70, n 11, pp. 1846-53.

Levinson Arik, 2016, «Are Energy Efficiency Standards Less Regressive Than Energy Taxes? », Working Paper.

Liu Jing-Yu, Shinichiro Fujimori et Toshihiko Masui, 2016, «Temporal and spatial distribution of global mitigation cost: INDCs and equity », Environmental Research Letters, vol. 11, n 11, 114004.

Lobell David B., Wolfram Schlenker et Justin Costa-Roberts, 2011, «Climate Trends and Global Crop Production Since 1980 », Science, vol. 333, $\mathrm{n}^{\circ} 6042$, pp.616-20. https://doi.org/10.1126/science. 1204531.

Logan John R., 2006, "The impact of Katrina: Race and class in stormdamaged neighborhoods ».

Masozera Michel, Melissa Bailey et Charles Kerchner, 2007, «Distribution of impacts of natural disasters across income groups: A case study of New Orleans », Ecological Economics, vol. 63, n² 2-3, pp. 299-306.

Matthews H. Damon, 2016, «Quantifying Historical Carbon and Climate Debts among Nations », Nature Climate Change, vol. 6, $\mathrm{n}^{\circ} 1$, pp. 60-64. https://doi.org/10.1038/nclimate2774.

Matthews H. Damon, Tanya L. Graham, Serge Keverian, Cassandra Lamontagne, Donny Seto et Trevor J. Smith, 2014, " National contributions to observed global warming ", Environmental Research Letters, vol. $9, \mathrm{n}^{\circ} 1,014010$.

Mellinger Andrew D., Jeffrey D. Sachs et John Luke Gallup, 2000, "Climate, coastal proximity, and development », The Oxford handbook of economic geography, $\mathrm{n}^{\circ} 169$, p. 194.

Milanovic Branko, 2016, Global Inequality: A New Approach for the Age of Globalization, Harvard University Press.

Moser Caroline, 2007, "Asset accumulation policy and poverty reduction", Reducing global poverty: The case for asset accumulation, pp. 83-103.

Myers Candice A., Tim Slack et Joachim Singelmann, 2008, « Social vulnerability and migration in the wake of disaster: the case of Hurricanes Katrina and Rita ", Population and Environment, vol. 9, $\mathrm{n}^{\circ}$ 6, pp. 271-91.

Nkedianye David, Jan de Leeuw, Joseph O. Ogutu, Mohammed Y. Said, Terra L. Saidimu, Shem C. Kifugo, Dickson S. Kaelo et Robin S. Reid, 2011, " Mobility and livestock mortality in communally used pastoral areas: the impact of the 2005-2006 drought on livestock mortality in Maasailand », Pastoralism: Research, Policy and Practice, vol. 1, $\mathrm{n}^{\circ} 1$, p. 17. 
Noack Frederik, Sven Wunder, Arild Angelsen et Jan Börner, 2015, "Responses to Weather and Climate: A Cross-Section Analysis of Rural Incomes », The World Bank Policy Research Working Papers. https:// doi.org/10.1596/1813-9450-7478.

Ohlendorf Nils, Michael Jakob, Jan Christoph Minx, Carsten Schröder et Jan Christoph Steckel, 2018, « Distributional Impacts of Climate Mitigation Policies - A Meta-Analysis », SSRN Scholarly Paper ID 3299337, Rochester, NY, Social Science Research Network. https:// papers.ssrn.com/abstract=3299337.

O’Neill Daniel W., Andrew L. Fanning, William F. Lamb et Julia K. Steinberger, 2018, "A good life for all within planetary boundaries », Nature Sustainability, vol. $1, \mathrm{n}^{\circ} 2, \mathrm{p} .88$.

Otto Ilona M., Kyoung Mi Kim, Nika Dubrovsky et Wolfgang Lucht, 2019, "Shift the focus from the super-poor to the super-rich », Nature Climate Change, vol. $9, \mathrm{n}^{\circ} 2$, p. 82 .

Pachauri Shonali, 2014, «Household electricity access a trivial contributor to CO 2 emissions growth in India », Nature Climate Change, vol. 4, $\mathrm{n}^{\circ} 12$, p. 1073 .

Pachauri Shonali, Bas J. van Ruijven, Yu Nagai, Keywan Riahi, Detlef P. van Vuuren, Abeeku Brew-Hammond et Nebojsa Nakicenovic, 2013, «Pathways to achieve universal household access to modern energy by 2030 », Environmental Research Letters, vol. 8, n 2, 024015.

Painter James, 2007, «Deglaciation in the Andean region », Human development report, 2008.

Pan Xunzhang, Hailin Wang, Ziwei Wang, Lu Lin, Qi Zhang, Xinzhu Zheng et Wenying Chen, 2019, "Carbon Palma Ratio: A New Indicator for Measuring the Distribution Inequality of Carbon Emissions among Individuals », Journal of Cleaner Production, n² 241, décembre, 118418. https://doi.org/10.1016/j.jclepro.2019.118418.

Park Jisung, Mook Bangalore, Stephane Hallegatte et Evan Sandhoefner, 2018, "Households and Heat Stress: Estimating the Distributional Consequences of Climate Change », Environment and Development Economics, vol. 23, n³, pp. 349-68. https://doi.org/10.1017/S1355770 X1800013X.

Parry Ian W. H., 2004, "Are Emissions Permits Regressive? », Journal of Environmental Economics and Management, vol. 47, $\mathrm{n}^{\circ} 2$, pp. 364-87. https://doi.org/10.1016/j.jeem.2003.07.001.

Patankar Archana, 2015, The exposure, vulnerability, and ability to respond of poor households to recurrent floods in Mumbai, The World Bank.

Peters Glen P., Jan C. Minx, Christopher L. Weber et Ottmar Edenhofer, 2011, « Growth in emission transfers via international trade from 1990 to 2008 », Proceedings of the national academy of sciences, vol. $108, \mathrm{n}^{\circ} 21$, 8903-8. 
Piketty T. et L. Chancel, 2015, "Carbon and Inequality: From Kyoto to Paris. Trends in the Global Inegality of Carbon Emissions (1998-2013) \& Prospects for an Equitable Adaptation Fund», Paris school of economics.

Piketty Thomas, Emmanuel Saez et Gabriel Zucman, 2018, « Distributional National Accounts: Methods and Estimates for the United States », The Quarterly Journal of Economics, vol. 133, $\mathrm{n}^{\circ} 2$, pp. 553-609. https:// doi.org/10.1093/qje/qjx043.

Pottier Antonin, Aurélie Méjean, Olivier Godard et Jean-Charles Hourcade, 2017, «A survey of global climate justice: from negotiation stances to moral stakes and back», International Review of Environmental and Resource Economics, vol. 11, $\mathrm{n}^{\circ}$ 1, pp. 1-53. https://doi.org/10.1561/ 101.00000090.

Quéré Corinne, Robbie Andrew, Pierre Friedlingstein, Stephen Sitch, Judith Hauck, Julia Pongratz, Penelope Pickers, Jan Ivar Korsbakken, Glen Peters et Josep Canadell, 2018, "Global carbon budget 2018 », Earth System Science Data, vol. 10, n 4, pp. 2141-94.

Rao Narasimha D., 2014, «International and Intranational Equity in Sharing Climate Change Mitigation Burdens », International Environmental Agreements: Politics, Law and Economics, vol. 14, n 2, pp. 129-46. https://doi.org/10.1007/s10784-013-9212-7.

Rao Narasimha D. et Paul Baer, 2012, « 'Decent living' emissions: a conceptual framework », Sustainability, vol. 4, n 4, pp. 656-81.

Rao Narasimha D. et Jihoon Min, 2018a, «Less global inequality can improve climate outcomes", Wiley Interdisciplinary Reviews: Climate Change, vol. $9, \mathrm{n}^{\circ} 2$, e513.

—, $2018 b$, «Decent Living Standards: Material Prerequisites for Human Wellbeing », Social Indicators Research, vol. 138, $\mathrm{n}^{\circ} 1$, pp. 225-44. https://doi.org/10.1007/s11205-017-1650-0.

Rao Narasimha D., Keywan Riahi et Arnulf Grubler, 2014, "Climate impacts of poverty eradication », Nature Climate Change, vol. $4, \mathrm{n}^{\circ} 9$, p. 749.

Rao Shilpa, Shonali Pachauri, Frank Dentener, Patrick Kinney, Zbigniew Klimont, Keywan Riahi et Wolfgang Schoepp, 2013, «Better air for better health: Forging synergies in policies for energy access, climate change and air pollution », Global Environmental Change, vol. 23, $\mathrm{n}^{\circ} 5$, pp. 1122-30.

Reeves Eleanor Krause et Richard V., 2017, « Hurricanes Hit the Poor the Hardest ", Brookings (blog). 18 septembre 2017. https://www. brookings.edu/blog/social-mobility-memos/2017/09/18/hurricaneshit-the-poor-the-hardest/.

Reguant Mar, 2018, « The Efficiency and Sectoral Distributional Impacts of Large-Scale Renewable Energy Policies », Journal of the Association of 
Environmental and Resource Economists, vol. 6, $\mathrm{n}^{\circ} \mathrm{S} 1$, pp. S129-68. https://doi.org/10.1086/701190.

Ritchie Hannah et Max Roser, 2017, « CO? and Greenhouse Gas Emissions », Our World in Data, mai. https://ourworldindata.org/co2-andother-greenhouse-gas-emissions.

Roberts J. Timmons, 2001, "Global Inequality and Climate Change», Society \& Natural Resources, vol. 14, n 6, pp. 501-9. https://doi.org/ 10.1080/08941920118490.

Robiou du Pont Yann, M. Louise Jeffery, Johannes Gütschow, Joeri Rogelj, Peter Christoff et Malte Meinshausen, 2017, «Equitable Mitigation to Achieve the Paris Agreement Goals ", Nature Climate Change, vol. 7, $\mathrm{n}^{\circ}$ 1, pp. 38-43. https://doi.org/10.1038/nclimate3186.

Rosenzweig Cynthia, Joshua Elliott, Delphine Deryng, Alex C. Ruane, Christoph Müller, Almut Arneth, Kenneth J. Boote et al., 2014, « Assessing Agricultural Risks of Climate Change in the 21st Century in a Global Gridded Crop Model Intercomparison", Proceedings of the National Academy of Sciences, vol. 111, n 9, pp. 3268-73. https://doi. org/10.1073/pnas.1222463110.

Scherer Laura, Paul Behrens, Arjan de Koning, Reinout Heijungs, Benjamin Sprecher et Arnold Tukker, 2018, "Trade-offs between social and environmental Sustainable Development Goals », Environmental science \& policy, $\mathrm{n}^{\circ} 90$, pp. 65-72.

Sen Amartya K., 1997, «From Income Inequality to Economic Inequality », Southern Economic Journal, vol. 64, n 2, pp. 384-401. https:// doi.org/10.2307/1060857.

Shue Henry, 1993, «Subsistence emissions and luxury emissions », Law \& Policy, vol. 15, n 1, pp. 39-60.

— 2019, "Subsistence protection and mitigation ambition: Necessities, economic and climatic", The British Journal of Politics and International Relations, vol. 21, $\mathrm{n}^{\circ} 2$, pp. 251-62.

Smith Kirk R., Howard Frumkin, Kalpana Balakrishnan, Colin D. Butler, Zoë A. Chafe, Ian Fairlie, Patrick Kinney et al., 2013, « Energy and Human Health », Annual Review of Public Health, vol. 34, n 1, pp. 159-88. https:/ /doi.org/10.1146/annurev-publhealth-031912-114404.

Sommer Mark et Kurt Kratena, 2017, « The carbon footprint of European households and income distribution ", Ecological Economics, $\mathrm{n}^{\circ} 136$, pp. 62-72.

Strömberg David, 2007, « Natural disasters, economic development, and humanitarian aid», Journal of Economic perspectives, vol. 21, $\mathrm{n}^{\circ} 3$, pp. 199-222.

Supit I., C. A. van Diepen, A. J. W. de Wit, P. Kabat, B. Baruth et F. Ludwig, 2010, "Recent changes in the climatic yield potential of various crops in Europe », Agricultural Systems, vol. 103, n 9, pp. 683-94. https:// doi.org/10.1016/j.agsy.2010.08.009. 
Taconet N., Méjean, A., Guivarch, C., 2020, «Influence of climate change impacts and mitigation costs on inequality between countries ", Climatic Change (forthcoming).

Tait Louise et Harald Winkler, 2012, «Estimating greenhouse gas emissions associated with achieving universal access to electricity for all households in South Africa", Journal of Energy in Southern Africa, vol. $23, \mathrm{n}^{\circ} 4$, pp. 8-17.

Vogt-Schilb Adrien, Brian Walsh, Kuishuang Feng, Laura Di Capua, Yu Liu, Daniela Zuluaga, Marcos Robles et Klaus Hubaceck, 2019, « Cash Transfers for Pro-Poor Carbon Taxes in Latin America and the Caribbean ", Nature Sustainability, vol. 2, n 10, pp. 941-48. https://doi.org/10.1038/ s41893-019-0385-0.

Ward D. S. et N. M. Mahowald, 2014, «Contributions of Developed and Developing Countries to Global Climate Forcing and Surface Temperature Change », Environmental Research Letters, vol. 9, n 7, 074008. https://doi.org/10.1088/1748-9326/9/7/074008.

Wiedenhofer Dominik, Dabo Guan, Zhu Liu, Jing Meng, Ning Zhang et YiMing Wei, 2017, "Unequal Household Carbon Footprints in China », Nature Climate Change, vol. 7, $\mathrm{n}^{\circ}$ 1, pp. 75-80. https://doi.org/10.1038/ nclimate3165.

Winkler Harald, Thapelo Letete et Andrew Marquard, 2013, «Equitable access to sustainable development: operationalizing key criteria », Climate Policy, vol. 13, $\mathrm{n}^{\circ} 4$, pp. 411-32. https://doi.org/10.1080/ 14693062.2013.777610.

«World's 15 Countries with the Most People Exposed to River Floods », 2015, World Resources Institute. 5 mars 2015. https://www.wri.org/ blog/2015/03/world-s-15-countries-most-people-exposed-river-floods. 


\section{Chapitres de rapports du GIEC}

GIEC, Cinquième rapport d'évaluation, Groupe 2, chapitre 13.

Olsson L., M. Opondo, P. Tschakert, A. Agrawal, S. H. Eriksen, S. Ma, L.N. Perch et S. A. Zakieldeen, 2014, "Livelihoods and poverty », in Climate Change 2014: Impacts, Adaptation, and Vulnerability. Part A: Global and Sectoral Aspects. Contribution of Working Group II to the Fifth Assessment Report of the Intergovernmental Panel on Climate Change [Field C. B., V. R. Barros, D. J. Dokken, K. J. Mach, M. D. Mastrandrea, T. E. Bilir, M. Chatterjee, K. L. Ebi, Y. O. Estrada, R. C. Genova, B. Girma, E. S. Kissel, A. N. Levy, S. MacCracken, P. R. Mastrandrea et L. L.White (eds.)], Cambridge University Press, Cambridge, United Kingdom et New York, NY, USA, pp. 793-832.

\section{GIEC, Rapport spécial 1.5, Chapitre 3.}

Hoegh-Guldberg O., D. Jacob, M. Taylor, M. Bindi, S. Brown, I. Camilloni, A. Diedhiou, R. Djalante, K. L. Ebi, F. Engelbrecht, J. Guiot, Y. Hijioka, S. Mehrotra, A. Payne, S.I. Seneviratne, A. Thomas, R. Warren et G. Zhou, 2018, «Impacts of $1.5^{\circ} \mathrm{C}$ Global Warming on Natural and Human Systems ", in Global Warming of $1.5^{\circ} \mathrm{C}$. An IPCC Special Report on the impacts of global warming of $1.5^{\circ} \mathrm{C}$ above pre-industrial levels and related global greenhouse gas emission pathways, in the context of strengthening the global response to the threat of climate change, sustainable development, and efforts to eradicate poverty [Masson-Delmotte V., P. Zhai, H.-O. Pörtner, D. Roberts, J. Skea, P. R. Shukla, A. Pirani, W. Moufouma-Okia, C. Péan, R. Pidcock, S. Connors, J. B. R. Matthews, Y. Chen, X. Zhou, M. I.Gomis, E. Lonnoy, T. Maycock, M. Tignor et T. Waterfield (eds.)]. In Press.

\section{GIEC, Rapport Spécial 1.5, Chapitre 5.}

Roy J., P. Tschakert, H. Waisman, S. Abdul Halim, P. Antwi-Agyei, P. Dasgupta, B. Hayward, M. Kanninen, D. Liverman, C. Okereke, P. F. Pinho, K. Riahi et A. G. Suarez Rodriguez, 2018, "Sustainable Development, Poverty Eradication and Reducing Inequalities » in Global Warming of $1.5^{\circ} \mathrm{C}$. An IPCC Special Report on the impacts of global warming of $1.5^{\circ} \mathrm{C}$ above preindustrial levels and related global greenhouse gas emission pathways, in the context of strengthening the global response to the threat of climate change, sustainable development, and efforts to eradicate poverty [Masson-Delmotte V., P. Zhai, H.-O. Pörtner, D. Roberts, J. Skea, P. R. Shukla, A. Pirani, W. Moufouma-Okia, C. Péan, R. Pidcock, S. Connors, J. B. R. Matthews, Y. Chen, X. Zhou, M. I. Gomis, E. Lonnoy, T. Maycock, M. Tignor et T. Waterfield (eds.)]. In Press. 